\title{
PERGAMINS DE L'ÈPOCA \\ DEL COMTE RAMON BORRELL DE BARCELONA, DE LA SECCIÓ DE CANCELLERIA DE L'ARXIU DE LA CORONA D'ARAGÓ
}

\author{
Josefina Font i Bayell
}

\section{INTRODUCCIÓ}

El motiu d'haver escollit els pergamins de l'època del comte Ramon Borrell per fer-ne un catàleg que pugui ajudar els estudiosos del segles x $\mathrm{i}$ xi ha estat el fet que anys enrera, el doctor Udina, aleshores director de l'Arxiu de la Corona d'Aragó, em va manifestar el seu desig de reunir els pergamins que fan esment de la ciutat i del territori de Barcelona, posteriors als ja publicats en el seu llibre El Archivo Condal de Barcelona.

Vaig començar a fer-ho però, per diferents raons, no vaig acabar la tasca començada. Ara m'ha semblat que era el moment oportú de dur-la a terme, en part perquè m'he limitat als pergamins del comte Ramon Borrell, però n'he donat el catàleg de tots, prescindint de fer la distinció entre els que fan referència a la ciutat o al territori, i els que afecten diferents indrets de Catalunya.

L'extensió limitada dels treballs en una Miscel-lània no em permet de fer altra cosa que donar a conèixer molt breument el contingut d'uns pergamins que pertanyen al moment històric de la formació de Barcelona i de Catalunya.

Una dificultat greu ha estat la catalanització dels noms llatins, tenint en compte que la llengua parlada no era pas l'escri- 
ta. Haig d'agrair a Jaume Riera i Sans uns consells encertats, la revisió del treball $\mathrm{i}$ el suggeriment que els noms femenins acabats en $o$ i que criden l'atenció actualment, cal accentuar-los, perquè encara en trobem algun vestigi en la llengua parlada, com és el cas dels noms Isabeló i Rosó. També correspon l'accent a la síl-laba final de noms masculins acabats en vocal. Com a particularitats onomàstiques he constatat que el levita Ugubertus, en dos pergamins hi trobo escrit Augbertus en el text, però ell signa sempre amb el nom d'Ubertus. Un nom: Paulus, faber, el trobo en el text de dos pergamins $i$ entre els signants de setze documents, amb una signatura molt característica. El nom de Vives apareix de moltes maneres, com Vivane, que caldria traduir per «Vivà». En aquest cas i en altres que es presenten, he cregut convenient unificar les formes diferents en la més corrent, quan es tracta del mateix personatge.

Quant a l'ordre cronològic, els he deixat en el mateix en què es troben dins les carpetes, $\mathrm{i}$, per tant, d'acord amb el donat pels arxivers del segle XIX que van fer una interpretació de la cronologia dels reis de França. Però tenint en compte l'estudi fet pel doctor A.M. Mundó pel que fa als anys del rei Robert, poso entre parèntesis l'any que els correspon d'acord amb aquesta nova datació.

Un pergamí interessant i que planteja un problema és el que té el número 92 que, per la intitulació, pertany al comte Ramon Berenguer I i a la comtessa Almodis. La menció que fa a Ramon Folc ho confirma, però la data de l'any tretzè del rei Robert i la signatura del bisbe Oliba d'Elna (1009-1013) va fer que fos catalogat entre els del comte Ramon Borrell. Segons l'opinió del doctor A.M. Mundó, a qui ho he consultat, el document és fals. Per la paleografia, i posant l'atenció en la forma o dibuix d'algunes lletres, és molt clar que va ser escrit en el segle xII; es tracta, doncs, d'una falsificació molt posterior. Agraeixo al doctor Mundó la seva aportació. Ningú que jo sàpiga no ha notat aquesta anomalia. Cal tenir en compte que aquest pergamí, copiat en el Liber Feudorum Maior - en un foli dels perduts en el segle XVII-, ha estat publicat per F. Miquel Rosell (Barcelona 1945) però amb la particularitat que en el text complet del vol. I, 
p. 216, la data és la de 31 de març de 1009 , mentre que en el resum en castellà del vol. II, p. 381, dóna la data de 31 de març de 1043, cosa del tot inexplicable, perquè la datació és ben clara, tant en el pergamí com en el text imprès.

El pergamí núm. 119, sense data, publicat també en el Liber Feudorum Maior, correspon al comte Ramon de Pallars a l'època en què estava casat amb la comtessa Ermessenda que, segons F. Valls i Taberner en Els origens dels comtats de Pallars i Ribagorça (Barcelona 1918), s'escauria cap a l'any 1029; per tant, no seria de l'època del comte Ramon Borrell de Barcelona. És possible que quan van ser ordenats aquests documents confonguessin els dos comtes, el de Barcelona i el de Pallars, per la coincidència dels noms de les seves esposes. També el publicà F. UDINA I Martorell: Sacramental romanceado de la primera mitad del siglo XI, a «B.R. Academia de Buenas Letras» XVII (1944) pp. 273-277.

Una altra dificultat, la trobo en el pergamí núm. 47, que també correspon a un comte Ramon de Pallars, que pot ser el mateix del pergamí 119, però la data és imprecisa. Hi llegim «... in mense augusto anno millesimum regnantem regem spectantem). $\mathrm{Si}$ volien dir que el document va ser fet en algun any posterior a l'any 1000, hauriem de suposar que ho fou l'any 1031, a la mort del rei Robert; per tant, tampoc no seria coetani del comte $\mathbf{R a}$ mon Borrell.

A més a més dels quatre pergamins publicats en el Liber Feudorum Maior, que són els números 39, 75, 92 i 119, ho ha estat el núm. 85 per Josefina SOLER GARcía en El cartulario de Tavèrnoles, Castelló de la Plana 1964, p. 49; i per Josep Nogués 1 Estany en la Història del monestir de Sant Sadurnt de Tavèrnoles, Barcelona 1973, p. 85. El pergamí núm. 27 ha estat reproduit a la Colectánea Paleográfica de la Corona de Aragón, a cura de Josefina i M. Dolors Mateu IBars, Barcelona 1980, làmina 10 . I el document núm. 106 ha estat estudiat $\mathrm{i}$ resumit per Josep M. SANS I TRAVÉ a Quaderns d'Història Tarraconense, I, 1977, p. 18. 


\section{PERGAMINS}

993 desembre 3

$N^{\circ} 1$

Marcús i la seva muller Eimena venen als esposos Vives i Ermovígia terres i vinyes en diferents indrets del territori de Barcelona, en els termes d'Or trobat, del Cogoll, de Sant Andreu i de Provençals. Assenyalen els límits del territori: al nord, el cim de les muntanyes; a l'est, el riu Besòs; a migdia, el mar; i a ponent, el riu Llobregat. Ho venen tot pel preu de set mancusos d'or cuit jafarí.

993 desembre 19

$\mathrm{N}^{\circ} 2$

El levita Ugubert ven als esposos Vives i Ermovígia una vinya en el suburbi de Barcelona, en el terme de Parets primes, pel preu de deu quarteres d'ordi corrent.

994 gener 29

$\mathrm{N}^{\circ} 3$

El comte Ramon [Borrell] i la comtessa Ermessenda venen al prevere Sanç un alou al comtat d'Osona, pel preu de sis unces d'or.

994 març 31

$\mathrm{N}^{\circ} 4$

Guifred i la seva muller Oruça donen a Bladerig una mujada de terra per plantar-hi o per edificar-hi, al comtat de Barcelona, a la vall de Torrelles.

994 maig 21

$\mathrm{N}^{\circ} 5$

Claudia ven al diaca Ató una vinya de quatre mujades, al comtat de Barcelona, al Vallès, en el lloc d'Olmedó, pel preu de dotze mancusos.

994 octubre $?$

$\mathrm{N}^{\circ} 6$

Els esposos Pau i Sunló venen a Vives, fill de Llorenç, unes terres situades en una illa del riu Besòs, en el terme del castell de Provençals, pel preu de dos mancusos d'or cuit legal.

995 gener 28

$\mathrm{N}^{\circ} 7$

Ermengarda ven al sacerdot Oriol la meitat d'un casal i d'una peça de terra al comtat d'Osona, a l'apendici del castell de Gurb, a la vila de Gurb, pel preu de deu sous. 
Arcedònia i les seves filles Cusca i Trasgonça venen als esposos Frugà i Tructelda una casa, compresos el terreny i l'edificació, i una mujada de terra, al territori de Barcelona, pel preu de sis sous i quatre mancusos.

995 abril 18

$\mathrm{N}^{\circ} 9$

Ugubert ven a Vives una terra al comtat de Barcelona, en el lloc anomenat Caldera [sic] del terme de Provençals, pel preu de sis mancusos d'or cuit jafarí.

995 abril 18

$\mathrm{N}^{\circ} 10$

Seniofred, a qui anomenen Navarro, ven a Vives una terra que posseeix en el territori de Barcelona, prop dels Arcs, pel preu d'una unça d'or cuit jafarí legal.

995 juny 17

$\mathrm{N}^{\circ} 11$

Bellida i Consalva venen a Olibà, comprador, diverses peces de vinya al comtat de Cerdanya, a la vall de Ribes, a la vila anomenada Brugera (Burguera).

995 juliol 25

$\mathrm{N}^{\circ} 12$

Eldefred i la seva muller Ermengarda venen als esposos Durand i Senior terres i vinyes al comtat de Barcelona, en el terme d'Olèrdola, a Vallmoll, pel preu de dues unces i quatre mancusos d'or.

995 agost 26

$\mathrm{N}^{\circ} 13$

Erinó, filla de Prexat, ven a Vives una vinya en el territori de Barcelona, en el terme del Besòs, pel preu de vint sous i una mesura i mitja d'ordi.

995 setembre 18

$\mathrm{N}^{\circ} 14$

Gotmar i la seva muller Ermengarda venen als esposos Segla i Gisclavara un casal situat en el burg de Barcelona, als Arcs antics, pel preu d'un mancús d'or cuit i d'un sou.

995 setembre 26

$N^{\circ} 15$

Els sacerdots Eribert, Miró i Barsand, marmessors testamentaris de Petroni, fan una carta de donació, a favor de l'abadessa Ingilberga, de l'alou que el difunt tenia a Vallfogona, a la vall de Ripoll, al comtat d'Osona. 
Aianric i la seva muller Guarengarde venen a Frugà i a Tructelda una casa i un hort amb els arbres i el pou, i l'heretat que és al comtat de Barcelona, al terme de Sant Adrià, pel preu de dues unces de bon or cuit jafari.

995 desembre 20

$N^{\circ} 17$

Eló, anomenada Bonadona, ven a Ferriol i a la seva muller Fremosa un camp d'un prat que posseeix en el territori de Barcelona, en el lloc anomenat «lo Bergadell», per cinquanta-cinc sous.

996 gener 2

$\mathrm{N}^{\circ} 18$

Isarn, Guillem, Salamó, el prevere Adalbert $\mathrm{i}$ els levites Giscafred i Teotfred, marmessors testamentaris de la comtessa Ermengarda, fan una escriptura de donació de l'alou que la comtessa tenia al Pedrer, a favor de la monja Espana.

996 gener 10

$\mathrm{N}^{\circ} 19$

El levita Augbert (Ugubert) ven al seu germà Vives i a la muller d'aquest, Ermovígia, unes terres en dos llocs del territori de Barcelona, en el terme de Provençals, en el lloc anomenat la Calvera, pel preu d'una unça d'or cuit.

996 febrer 17

$N^{\circ} 20$

Una dona, Ranló, ven a Ermenard, anomenat Marcús, una vinya erma, al comtat de Barcelona, al Callès [sic], a la vila «Pugnoso», pel preu de quatre sous.

996 març 15

$\mathrm{N}^{\circ} 21$

Gondemar, fill de Teuderic, ven a Vives un hort amb arbres i un pou, fora dels murs de Barcelona, pel preu de dos mancusos i la quarta part d'un altre, d'or cuit i net.

996 abril 13

$N^{0} 22$

Ermelda, abadessa de Sant Pere de les Puel-les, de Barcelona, amb les seves monges, permuten unes terres amb Guimarà. Les monges donen una terra en el terme de Sant Andreu, prop de la parròquia, a canvi d'una altra terra en el lloc d'Or trobat. 
El prevere Ballfred ven a Ennegó, anomenat Bonfill, una terra a Vallmoll, en el terme d'Olèrdola, al comtat de Barcelona, pel preu de dos mancusos d'or.

996 maig 20

Bonfill, fill de Sendered, conegut també per Ennegó, ven a Matfred una terra en el lloc de Vallmoll, en el terme d'Olèrdola, al comtat de Barcelona, pel preu de dos mancusos d'or.

996 juliol 22

$N^{\circ} 25$

Labadessa Ermelda i les monges del cenobi de Sant Pere [de les Puel-les] fan una permuta amb Vives, fill del difunt Llorenç. Les monges donen una terra del territori de Barcelona, a l'illa del riu Besòs, $i$ a canvi reben una vinya $i$ dos mancusos d'or cuit $i$ net.

\section{6 agost 3}

$\mathrm{N}^{\circ} 26$

Ugubert i la seva muller Espetosa, venen als esposos Vives i Ermovígia una terra a l'illa del Besòs, en el terme de Provençals, al comtat de Barcelona, pel preu de setze mancusos d'or cuit i net.

\section{6 agost 10}

$\mathrm{N}^{\circ} 27$

Ugubert ven al seu germà Vives una terra i una vinya al lloc anomenat la Calvera, a Provençals, al comtat de Barcelona, pel preu de tres pesades (monedes) d'argent. (Reproduit a CPCA, làmina 10).

996 agost 31

$\mathrm{N}^{\circ} 28$

Una dona, Senior, ven al seu marit Durand unes terres i vinyes a Vallmoll, en el terme d'Olèrdola, al comtat de Barcelona, pel preu d'una unça d'or.

996 setembre 3

$N^{\circ} 29$

Indaleci i la seva muller Saborida fan una permuta amb Orús. Els primers donen una vinya en el terme de Marunyà, al comtat de Barcelona, a canvi d'una terra i de dos mancusos d'or.

997 gener 18

$\mathrm{N}^{\circ} 30$

Els comtes Ramon [Borrell] i Ermessenda venen als esposos Rigol i Quixel un prat en el terme de Vilalba, al comtat de Barcelona. 
Centurió i la seva muller Bella venen al diaca Ató una peça de terra al comtat de Barcelona, al Vallès, en el lloc d'Olmedó, pel preu de sis sous.

997 maig 31

Una dona, Frugó, i el seu fill Audegari, venen als esposos Frugel i Teruntel una terra amb un casal, al comtat de Barcelona, en el terme de Tiano [Ticià], pel preu de dues unces d'or jafarí.

997 (996) juliol 29

El vescomte Seniofred ven a Radulf uns alous al comtat d'Osona i la vila anomenada Orsal i la Vila-Rubia, pel preu de dos-cents sous.

997 desembre 28

$\mathrm{N}^{\circ} 34$

Amaltrud i el seu fill Guillem, vescomte d'Empúries, i els seus germans Bligeri i Sunyer venen a Vives, fill de Llorenc, una terra de dues mujades, al comtat de Barcelona, en el terme de Provençals, a les illes del riu Besòs, per dues unces d'or cuit, pesades fidelment.

998 gener 21

$\mathrm{N}^{\circ} 35$

Escriptura d'empenyorament d'una parellada de terra situada en el territori de Barcelona, en el terme de Provençals, a la Torre Blanca. Una dona, Riquilda, es compromet a lliurar un cafís d'ordi per l'esmentada parellada a Sanç.

998 abril 19

$\mathrm{N}^{\circ} 36$

Sesenand ven al jutge Orús una vinya, en el territori de Barcelona, en el terme de Parets primes o d'Or trobat, prop de la Torre Blanca, per vuit mancusos d'or cuit.

998 maig 3

$\mathrm{N}^{\circ} 37$

Ermengarda, anomenada Bonafilla, filla del difunt Sisuald, ven a Angobert la part que li correspon d'una vinya situada al comtat de Barcelona, en el terme de Provençals, a les Parets primes, per un mancús d'or cuit de pes legal.

998 juny 9

$\mathrm{N}^{\circ} 38$

El levita Augbert (Ugubert) ven al seu germà Vives una vinya prop de la ciutat de Barcelona, en el terme de Parets primes, prop de la Torre Blanca, pel preu de cinc mancusos i mig d'or cuit jafarís de pes legal. 
Radulf ven a Adaleç, compradora, uns alous al comtat d'Osona o en els seus termes, és a dir, la vila d'Orsal i la Vila-Rubia, per doscents sous. (Publicat en el LFM, vol. I, p. 460).

998 (997) agost 26

El levita Ugubert ven a Vives una vinya en el territori de Barcelona, en el terme de Parets de Guisaliarda, per sis mancusos d'or cuit de moneda corrent.

998 novembre 1

El levita Ugubert ven als germans Petró, Sunyer i Gotmar una terra prop de la ciutat de Barcelona, en el terme de Provençals, pel preu d'una unça d'or cuit $\mathrm{i}$ net de pes legal.

999 (998) setembre 1

$N^{\circ} 42$

El levita Ugubert ven a Vives una terra en el territori de Barcelona, en el terme de Provençals, pel preu de vint- $i$-una quarteres d'ordi $i$ una quartera de blat de mesura legal.

1000 gener 10

$\mathrm{N}^{\circ} 43$

Trasllat d'una escriptura del comte i marquès Ramon i de la seva muller la comtessa Ermessenda. Venen a Hug, fill de Riquilda, un alou al comtat de Barcelona, en el terme del castell de Cervelló, pel preu de trenta unces d'or cuit jafarís.

1000 febrer 1

$\mathrm{N}^{\circ} 44$

Teuderic i la seva muller Alió venen als esposos Frugà i Tructelda una terra al comtat de Barcelona, a Tiano, pel preu de quatre mancusos d'or de moneda corrent.

1000 març 3

$N^{\circ} 45$

El comte i marquès Ramon ven a Bonhome, fill de Guisand, unes terres i vinyes en el territori de Barcelona, en el lloc de Mogòria, pel preu de nou unces i dos mancusos d'or cuit i net de pes legal. El comte posseỉa les terres per successió hereditària dels jueus morts [intestats] a la ciutat de Barcelona.

1000 març 12

$N^{\circ} 46$

Olibà ven a Bernat cinc peces de vinya al comtat de Cerdanya, a 
la vall de Ribes, a la vila de Brugera (Burguera), pel preu de vint (?) o l'equivalent en espècie.

1000 (?) agost

El comte Ramon [de Pallars] ven als esposos Ató i Ava una vinya "Estiva» al comtat de Ribagorça, en el lloc anomenat Estivella. Els compradors havien donat a canvi un cavall negre avaluat en tres unces. (V. Introducció).

1000 abril 25

$\mathrm{N}^{\circ} 48$

Onrada, consagrada a Déu, i els seus fills Pere i Teudebert, venen als esposos Olibà i Filmera una terra en el suburbi de Barcelona, en el lloc anomenat el $\mathrm{Pi}$, prop de la casa de Santa Maria, pel preu de nou mancusos i mig d'or cuit de pes legal.

1000 maig 10

$N^{\circ} 49$

Guisalmar i la seva muller Rihcell venen a Vives una terra en el terme de Provençals, pel preu d'un mancús d'or cuit.

1000 juny 1

$N^{\circ} 50$

Orús i la seva muller Gondemara venen als esposos Gotmar i Kasta una peça de terra en el territori de Barcelona, en el terme de Banyols, per un mancús i mig d'or de moneda corrent.

1000 novembre 21

$N^{\circ} 51$

El levita Ugubert ven al seu germà Miró una peça de terra prop de la ciutat de Barcelona, en el terme de Provençals, pel preu de (malmès) mancusos d'or cuit i net pesats legalment.

1000 desembre 1

$\mathrm{N}^{\circ} 52$

El levita Ugubert ven al seu germà Vives una vinya prop de la ciutat de Barcelona, en el terme de Provençals, en el lloc anomenat Camp de la Mula, pel preu de quinze mancusos i mig d'or cuit i net, pesats legalment.

1000 desembre 23

$\mathrm{N}^{\circ} 53$

El comte i marquès Ermengol [d'Urgell] ven a Guitard el castell de Pinell, al comtat d'Urgell, a la Marca, pel preu de deu unces d'or de moneda corrent. 
1001 (1000) agost 28

Sala ven a Ermengol i als seus fills Brocard i Sendra unes terres i vinyes conreades $i$ ermes, i cases amb la Torre Franca (?), al comtat de Manresa, en el castell d'Òdena, en el lloc anomenat Ardebell (afronta amb (astellfollit), pel preu de cinquanta unces d'or de mancusos vells.

1001 (1000) octubre 14

$N^{\circ} 55$

Sigirand i la seva muller Denna venen a Sanç Cortubi i a la seva muller Domeva dues peces de vinyes en dos llocs del territori de Barcelona, en el terme de Sants, pel preu d'una mancusada.

1001 (1000) octubre 19

$\mathrm{N}^{\circ} 56$

Una dona, Egó, filla de Sala, ven a Vives una cort, en el burg de la ciutat de Barcelona, pel preu de dos mancusos d'or cuit de pes legal.

1001 octubre 31

$\mathrm{N}^{\circ} 57$

Roderad i la seva muller Gisclavara venen als esposos Unifred i Eló una terra conreada al comtat de Barcelona, a Castellví, en el lloc anomenat Lorint.

1001 desembre 1

$N^{\circ} 58$

Ugubert, levita, ven al seu germà Vives una vinya amb els arbres que hi ha plantats, en el terme de Provençals, prop de la ciutat de Barcelona, pel preu d'onze mancusos d'or cuit i net legalment pesats.

1002 gener 1

$\mathrm{N}^{\circ} 59$

Una dona, Eldesenda, ven al jutge Orús una vinya al comtat de Barcelona, a Or trobat, pel preu de quatre mancusos d'or cuit i net legalment pesats.

1002 gener 16

$\mathrm{N}^{\circ} 60$

Guillem ven a Landeric un molí al comtat de Barcelona, al terme de Terrassa, en el lloc anomenat Castellar [del Vallès], pel preu de dos mancusos d'or.

1002 febrer 6

$N^{\circ} 61$

Asenar fa una carta de donació a favor de la seva filla Eló, monja, d'uns alous al comtat d'Osona, a la vall de Ripoll, que comprenen un mas en el lloc anomenat Torrent i un altre a la vila Ennegós. 
Abelló i la seva germana Maria venen a Sanç i a la seva muller Dominica tres peces de vinya en dos llocs del territori de Barcelona, en el terme de la vila de Sants, per un mancús d'or cuit de pes legal i sis sous de diners corrents.

1002 març 28

Ferriol i la seva muller Fermosa i els esposos Morató i Audesenda venen a Vives, fill del difunt Llorenç, una terra amb una casa i tots els seus annexos, situada en el territori de Barcelona, en el terme de Banyols, pel preu de tres unces d'or cuit de pes legal.

1002 (1001) setembre 24

$N^{\circ} 64$

El prevere Baldofred i una dona, Quillió, fan donació a l'església de Sant Pere Apòstol d'unes terres, d'unes vinyes, amb cases i horts etc. al comtat de Barcelona, en el lloc anomenat Duodecimo (Sant Pere de Romaní, a Molins de Rei).

1002 novembre 21

$\mathrm{N}^{\circ} 65$

El levita Ugubert i una dona, Guisla, venen a Vives una terra en el territori de Barcelona, en el terme de Provençals, pel preu de deu mancusos d'or cuit de pes legal.

1003 juny 7

$\mathrm{N}^{\circ} 66$

Bonfill ven als esposos Frugà i Tructelda dues peces de terra al comtat de Barcelona, en el terme de Tiano, pel preu de vuit mancusos.

1003 (1002) juliol 1

$\mathrm{N}^{\circ} 67$

Els esposos Altemir i Levegod venen a Frugá dues mujades i mitja de terra, al comtat de Barcelona, en el terme de Tiano, pel preu de nou mancusos de bon or cuit $\mathrm{i}$ net.

1003 octubre 1

$\mathrm{N}^{\circ} 68$

Els esposos Guitard i Garsenda venen a Guimarà una terra d'una mujada i més al comtat de Barcelona, en el terme de la Perusa.

1003 novembre 6

$\mathrm{N}^{\circ} 69$

El levita Ugubert ven al seu germà Vives una terra prop de Barcelona, a la vila de Provençals, en el lloc anomenat la Calvera, pel preu de sis mancusos d'or cuit legalment pesats. 
El levita Ugubert ven al seu germà Vives una casa al comtat de Barcelona, a la vila de Provençals, pel preu de cinc mancusos d'or cuit i un sac d'ordi.

1004 febrer 7

El prevere Hendelec $\mathrm{i}$ Vives venen a Pau, artesà, en compliment del testament de Bonhome, la casa d'aquest amb tots els annexos, en el territori de Barcelona, dins de la vila de Provençals, pel preu de cinc mancusos d'or.

1004 febrer 16

$\mathrm{N}^{\circ} 72$

Bonhome i la seva muller Guisul venen a Sanç i a la seva muller Dominica dues peces de terra en dos llocs del territori de Barcelona, en el terme de la vila de Sants, pel preu d'un mancús d'or cuit de pes legal.

1004 març 22

$\mathrm{N}^{\circ} 73$

El levita Ugubert i una dona, Guisla, venen a Vives una cort amb dos colomars, un pou etc. en el territori de Barcelona, a la vila de Provençals, pel preu de set mancusos d'or cuit de pes legal.

1004 (1003) agost 26

$\mathrm{N}^{\circ} 74$

Ansulf $\mathrm{i}$ la seva muller Emmó venen a la vescomtessa Adaleç cases i terres al comtat d'Osona, en el castell Orsalità (Orsal-Malla), en el terme de Puig-oriol, pel preu de tres mancusos i un sac d'ordi o l'equivalent en espècie.

1004 (1003) octubre 23

$\mathrm{N}^{\circ} 75$

Adroer i la seva muller Bellúcia permuten amb Adaleç una terra per una vinya al comtat d'Osona, a l'apendici Orsalità (Orsal-Malla), en el lloc anomenat Lodares. Trasllat posterior de 9.4.1057. (Publicat en el LFM, vol. I, p. 461).

1005 març 28

$\mathrm{N}^{\circ} 76$

Ermessenda ven a Vives una casa, un corral i un hort, a la vila de Provençals, al comtat de Barcelona, pel preu de vint-i-tres sous de diners de moneda corrent. 
El comte Bernat [Tallaferro] i la seva muller la comtessa Tota o Adaleç venen a Ardraan la meitat d'un alou al comtat d'Osona, en el lloc anomenat Lasens, excepte el castell, pel preu de cent cinquanta sous. Trasllat posterior de 17.6.1215.

\section{5 desembre}

Olibà empenyora al comte Bernat [Tallaferro] un alou que fou d'Ardeman i de la seva muller Llobatona al comtat d'Osona, a la vall de Ripoll, a la vila Karcases $i$ adjacents, pel preu de dos-cents sous.

1005 desembre 16

$\mathrm{N}^{\circ} 79$

Goitred i la seva muller Ricarda trameten la seva filla Emma a l'abadessa Ingilberga, del monestir de Sant Joan de les Abadesses, i donen com a dot un alou al comtat d'Osona, a la vall de Ripoll, a la vila anomenada Tenes, o en altres llocs dins els termes de Ripoll, en els comtats de Girona i d'Empúries, i en el comtat de Peralada.

1006 (1005) agost 3

$N^{\circ} 80$

El levita Ugubert ven a Ató una terra prop de la ciutat de Barcelona, en el terme d'Or trobat, pel preu de dos mancusos d'or.

1006 desembre 2

$\mathrm{N}^{\circ} 81$

Bellid i la seva muller Adaleda venen als esposos Vives i Ermovigia un casal amb una conducció d'aigua i una claveguera, pel preu de dos mancusos d'or cuit.

1006 desembre 13

$\mathrm{N}^{\circ} 82$

Gontar dóna a la casa de Sant Martí, al comtat berguedà, a la serra, una peça de vinya en el lloc de Puig-reig.

1007 gener 18

$N^{\circ} 83$

Ermemira ven a Teudiscle unes terres i unes cases al comtat de Barcelona, en el terme de... en el lloc anomenat Vall-bovera, pel preu de dos mancusos.

1007 febrer 3

$\mathrm{N}^{\circ} 84$

Seniofred i la seva muller Riquilda empenyoren a Vives tres mujades de terra en el territori de Barcelona, en el lloc anomenat Clerà, a la Torre Blanca, pel preu de vuit mancusos i quart d'or jafari, i es 
comprometen a tornar aquesta quantitat a la propera festa de Sant Cugat màrtir.

1007 juny 13

$\mathrm{N}^{\circ} 85$

El comte Ermengol [d'Urgell] fa una carta de donació, a favor del cenobi de Sant Sadurni [de Tavèrnoles], situat al comtat d'Urgell prop del riu Valira, d'un cens que comprèn totes les terres de la vall d'Andorra. En dóna els límits: a l'orient, el comtat de Cerdanya; al migdia, el riu Riuner; a l'occident, el comtat de Pallars; i al nord, el terme de Sabartés (al Capcir). També cedeix al monestir la meitat de tots els drets d'hostatge. (V. Introducció).

1007 (1006) octubre 14

$\mathrm{N}^{\circ} 86$

Viscafred i la seva muller Maria venen als esposos Sanç i Dominica unes cases, un corral i un pou, en el burg fora dels murs de la ciutat de Barcelona, prop dels Arcs, pel preu de set mancusos i mig d'or cuit de pes legal.

1007 novembre 7

$N^{\circ} 87$

Bellid i la seva muller Adaleç empenyoren a Regiat unes cases, un corral, unes terres, unes vinyes i uns arbres en el territori de Barcelona, a Provençals, pel preu de cinc unces, dos mancusos i la tercera part d'un mancús.

1007 desembre 13

$\mathrm{N}^{\circ} 88$

Bellid i Adaleç venen a Vives i a Guimarà unes terres i unes vinyes (no especifiquen el lloc), pel preu de vuit unces i quatre mancusos d'or.

1007 desembre 18

$N^{\circ} 89$

Guifred i la seva muller Vilea venen als esposos Alió i Onrada una peça de terra al comtat de Barcelona, al Vallès, a Lliçà d'Amunt, en el lloc anomenat Camp d'Oliva, pel preu de tres sous.

1008 (1007) desembre 13

$\mathrm{N}^{\circ} 90$

Ademar, anomenat també Seniofred, i la seva muller Riquilda, filla del difunt Eldefred, venen als esposos Vives i Ermovigia unes terres i la torre anomenada Torre Blanca, en el territori de Barcelona, en el lloc de Clerà, prop de les Parets antigues, pel preu de tres unces d'or i quatre mancusos. 
Adroer i la seva muller Bellúcia permuten amb el vescomte Seniofred i la seva muller, la vescomtessa Adaleç, una casa i unes terres a l'apendici del castell Orsalità (Orsal-Malla) i donen també un mancús d'or cuit, pel preu d'un altre camp que havien permutat abans.

El comte Ramon Berenguer i la comtessa Adalmur (Almodis) donen a Ramon Folc i a la seva muller Ermessenda, vescomtessa, la meitat de l'alou de Vallfornés que és a la parròquia de Santa Maria de Tagamanent. (Publicat en el LFM, vol. I, p. 216. Falsificació del s. XII. V. Introducció).

Teudelinda, abadessa del monestir de Sant Pere de les Puel-les, i totes les monges, amb assentiment del bisbe de Barcelona [Aeci] $\mathrm{i}$ amb decret del comte Ramon i de la comtessa Ermessenda, fan una permuta amb Guimarà. Labadessa $\mathrm{i}$ les monges donen un alou del monestir on aquell havia edificat una casa, en el territori de Barcelona, a Provençals. Elles reben, a canvi, unes vinyes en dos llocs, una al Cogoll, prop del cenobi, i unes altres de dues mujades i mitja a Provençals, prop de l'alou que elles donen; i també acusen rebut d'una suma de diners, o sigui, d'onze mancusos d'or i onze exarachellos (monedes) d'argent destinats a la restauració de l'esmentat cenobi.

Trasegòncia, viuda de Sendered, el seu fill Maier i la seva filla Ermel venen a Olibà suder una terra en el territori de Barcelona, en el terme del Cogoll antic, pel preu de quatre mancusos d'or.

1009 (juny/juliol)

Els esposos Truitari i Garsenda venen a Fruià, sacerdot, unes terres, amb les cases $\mathrm{i}$ els horts, que tenen al comtat de Manresa: al castell d'Oló, a Terraçola i a la vall «Posticiosa».

1009 (1008) agost 26

$\mathrm{N}^{\circ} 96$

Ansulf $i$ la seva muller Emmó venen a la vescomtessa Adaleç unes cases $i$ adjacents $i$ en un altre lloc una peça de terra al comtat d'Osona, en el castell Orsalità (Orsal-Malla), en el terme de Puig-oriol, pel preu de tres mancusos i un sac d'ordi. 
1009 (1008) setembre

Seniol, fill del difunt Bernat, i la seva muller Ermessenda, filla del difunt Guiscafred, venen a Vives, fill del difunt Llorenc, tres mujades i mitja de vinyes, en el territori de Barcelona, en el lloc anomenat la Perella, prop de la parròquia de Sant Andreu apòstol, pel preu de vinti-tres mancusos i mig d'or.

1010 (1009) juliol 23

$\mathrm{N}^{\circ} 98$

Morgades i la seva muller Godevira venen a Gilberga, abadessa, un clos de terra al comtat d'Osona, a la vall de Ripoll, pel preu de sis sous.

1011 març 13

$\mathrm{N}^{\circ} 99$

Guifred i la seva muller Guilia donen al [monestir] de Sant Marti, construït a l'illa Gallinera (en el golf de Gènova), el cenobi de Sant Pere de Riudebitlles amb els seus termes; alous i vinyes dels termes de Mediona i de Terraçola; aigües; el castell de Cabra, construït sobre la roca; i el seu propi castell. Fan un vitalici de dues unces d'or anual per la festa de sant Martí. Trasllat de 1198.

1012 juny 12

$\mathrm{N}^{\circ} 100$

Ansemund i la seva muller Orúcia venen a Olibà suder una vinya en el territori de Barcelona, en el terme del Cogoll antic, pel preu de cinc unces $i$ quatre mancusos d'or.

1012 juny 13

$\mathrm{N}^{\circ} 101$

Bernat i la seva muller Ermetruda venen a Ledgarda una terra amb arbres al comtat de Barcelona, al Vallès, dintre de la vila de Sentmenat, pel preu de quatre mancusos.

1012 (1011) agost 13

$N^{\circ} 102$

Ledgarda, monja de Sant Joan [de les Abadesses], fa una escriptura de donació a favor del monestir de Sant Joan Baptista d'un alou que tenia en el comtat de Vallespir.

1012 desembre 6

$\mathrm{N}^{\circ} 103$

Bonhome ven a Miró una vinya situada en dos llocs del comtat de Barcelona, en el terme de Sentmenat, a la «capella», pel preu de quatre mancusos d'or.

1013 maig 18

$N^{\circ} 104$

El comte Ramon i la seva muller Ermessenda donen un prat, en el 
terme de Vilalba, al Vallès, als veïns de la població perquè havien estat perjudicats en els seus drets.

1013 juny 14

$\mathrm{N}^{\circ} 105$

Seniofred i la seva muller Ermetruda venen a Miró unes cases en el comtat de Barcelona, al Vallès, a la vall de Sentmenat.

1013 (1012) juliol 10

$\mathrm{N}^{\circ} 106$

Sala i la seva muller Quintuló, anomenada Llobeta, venen al bisbe Borrell el castell de Santa Perpètua amb la seva parròquia i el seu terme; i el castell de Barberà amb els seus termes, pel preu de cent unces d'or.

1013 desembre 19

$N^{\circ} 107$

Publicació sagramental del testament del difunt Cristià, feta a l'altar de Sant Pancraç de l'església del Pi de Barcelona. Els marmessors manifesten la voluntat del difunt que deixa un alou i unes cases a la seva esposa, i als fills els llega en vida dues vaques i dues mujades de vinya, al Cogoll, a canvi del pagament de la dècima anual al cenobi de Sant Pere de les Puel-les.

1014 gener 9

$\mathrm{N}^{\circ} 108$

Bellús, fill del difunt Sabat, ven a Maria, viuda del jutge Orús, un hort amb arbres i un pou, un tros de terra i els edificis que hi són construits, en el burg extramurs de la ciutat de Barcelona, prop del Cogoll, pel preu de cinc mancusos d'or corrent de pes legal i una quantitat d'ordi.

1014 febrer 7

$\mathrm{N}^{\circ} 109$

Orús i la seva muller Geriberga venen a Ermovígia tres peces de terra en tres llocs del comtat de Barcelona, al Vallès, en el terme de la Roca, en el lloc anomenat el Mogent, pel preu d'una unça d'or cuit.

1014 març 31

$N^{\circ} 110$

Leguard $\mathrm{i}$ la seva muller Frug venen a Issarn quatre peces de vinya al comtat de Manresa, a Castelloli, pel preu de dos mancusos.

1014 octubre 25

$\mathrm{N}^{\circ} 111$

Olibà i la seva muller Cixol venen a la senyora Elliarda cinc quarteres de vinya amb arbres fruiters i no fruiters, al comtat de Barcelona, 
en el terme de Cervelló, al confí de Penyafel, en el lloc anomenat «les manyanes", per un cafís i tres sacs d'ordi.

1014 novembre 10

$\mathrm{N}^{\circ} 112$

Reifred, prevere, dóna a Sant Martí de Puig-reig una peça de terra i una altra de vinya.

1015 maig 4

$\mathrm{N}^{0} 113$

Miró, prevere, fill del difunt Bonhome, empenyora al prevere Aventí una peça de terra en el terme de l'Estany; un casal i un hort fora de les muralles de Barcelona, a la part del Regomir; dues peces de vinya en llocs diferents del territori de Barcelona. Reconeix que ha rebut dues unces $\mathrm{i}$ un mancús.

1016 març 1

$\mathrm{N}^{\circ} 114$

El bisbe Deodat reconeix la possessió d'unes terres, en el lloc de Cubelles del terme de Trullols, a una dona anomenada Emmó que les havia rebudes del bisbe Vives, difunt.

\section{6 abril 8}

$\mathrm{N}^{\circ} 115$

Gontuad i la seva muller Clàudia venen a Llobet una terra conreada i erma, al comtat de Barcelona, en el terme de Castellví, pel preu de cinc sous de diners.

1016 juny 1

$\mathrm{N}^{\circ} 116$

Trasver i la seva muller Guisla, i els esposos Vives i Guinidilda, venen a Borrell, fill de Guimara, una vinya en el territori de Barcelona, en el terme de la Perella, pel preu de setze mancusos.

1016 (1015) juliol 8

$\mathrm{N}^{\circ} 117$

Gotcefred ven a la monja Eló un alou al comtat d'Osona, a la vall de Ripoll, en el lloc anomenat Torrent o a la vila "Ennegó», pel preu de cinc mancusos d'or òtim.

Sense data

$\mathrm{N}^{\circ} 118$

El comte Gilabert i el seu fill Gaufred empenyoren uns masos de la vila Manyols a Ponç Bliger i a la seva muller Veliard, per cent sous de «rossets mascles». Quan li ho tornin, les monedes no poden ser inferiors a quaranta-quatre sous per lliura de plata, i el termini de la penyora va d'un Nadal a l'altre. 
Sense data [... 1047]

Jurament de fidelitat de Radulf Oriol al comte Ramon [de Pallars] i a la comtessa Ermessenda, pels castells d'Areny i d'Orrit. (Publicat al $L F M$, vol. I, p. 137. V. Introducció).

\section{ÍNDEX ONOMẢSTIC I TOPONIMIC}

Abelló, 62.

Adalec, 39, 75, 87, 88; -comtessa, 77; -vescomtessa, 74, 91, 96.

Adaleda, 81 .

Adalbert, prevere, 18.

Adalmur (Almodis), comtessa, 92.

Ademar, 90.

Adroer, 75, 91.

Aianric, 16.

Alió, 89.

Alió dona, 44.

Altemir, 67.

Amaltrud, 34.

Andorra, vall, 85 .

Ansemund, 100.

Ansulf, 74, 96.

Ardebell, 54.

Ardeman, 78.

Ardraan, 77.

Arcedònia, 8.

Arcs, 10, 86; -antics, 14.

Areny, castell, 119.

Asenar, 61.

Ató, 47, 80; -diaca, 5, 31.

Audegari, 32.

Audesenda, 63.

Angobert, 37.

Augbert, V. Ugubert.

Ava, 47. 
Aventí, prevere, 113.

Baldofred, prevere, 64 .

Banyols, 50, 63.

Barberà, castell, 106 .

Barsand, 15.

Bella, 31.

Bellfred, 23.

Bellid, 81, 87, 88.

Bellida, 11.

Bellúcia, 75, 91.

Bellús, 108.

Bergadell, 17.

Bernat, 46, 97, 101; -comte, 77, 78.

Besòs, riu, 1, 6, 25, 26, 34.

Bladerig, 4.

Bligeri, 34.

Bonfill, 23, 24, 66.

Bonafilla, 37.

Bonadona, 17.

Bonhome, 45, 71, 72, 103, 113.

Borrell, 116.

Barcelona, bisbe, 93.

Borrell, bisbe, 106.

Brugera (Burguera), 46.

Cabra, castell, 99.

Caldera (?), Calvera, 9, 19, 27, 69.

Callès, 20.

Camp de la Mula, 52.

Camp d'Oliva, 89.

Castellar [del Vallès], 60.

Castellfollit, 54.

Castellolí, 110.

Castellví, 57, 115.

Centurió, 31.

Cerdanya, comtat, 11, 46, 85.

Cervelló, 111; -castell, 43.

Cixol, 111. 
Clàudia, $5,115$.

Clerà, 84,90 .

Cogoll, 1, 93, 107, 108, antic, 94, 100.

Consalva, 11.

Cristià, 107.

Cubelles, lloc, 114.

Cusca, 8.

Denna, 55.

Deodat, bisbe, 114.

Domeva, 55.

Dominica, 62, 72, 86 .

"Duodecimo» [Sant Pere de Romaní], 64.

Durand, 12, 28.

Egó, 56.

Eimena, 1.

Eldefred, 12, 90.

Eldesenda, 59.

Eló, 17, 57; -monja, 61, 117.

Elliarda, 111.

Emma, 79.

Emmó, 74, 96, 114.

Empúries, 34, 79.

Ennegó, 23.

«Ennegós», vila, 61, 117.

Eribert, 15.

Erinó, 13.

Ermelda, abadessa, 22, 25.

Ermemira, 83.

Ermenard, 20.

Ermengarda, 7, 12, 14, 37; -comtessa, 18.

Ermengol, 54; -comte, 53, 85.

Ermessenda, 76, 97; -comtessa, 3, 30, 43, 93, 104;

-[de Pallars], 119; -vescomtessa, 92.

Ermetruda, 101, 105.

Ermel, 94.

Ermovígia, 1, 2, 19, 81, 90, 109.

Espana, monja, 18. 
Espetosa, 26.

Estany, terme, 113.

Estivella, 47.

«Estiva), 47.

Ferriol, 17, 63.

Filmera, 48.

Fremosa, 17, 63.

Frug, 110.

Frugà, $8,16,44,66,67$.

Frugel, 32 .

Frugó, 32.

Fruià, sacerdot, 95 .

Gallinera, illa, 99.

Garsenda, 68, 95.

Gaufred, 118.

Geriberga, 109.

Gilabert, 118 .

Gilberga, abadessa, 98 .

Girona, comtat, 79.

Gisclavara, 14, 57.

Giscafred, levita, 18.

Godevira, 98.

Goltred, 79.

Gondemar, 21.

Gondemara,50.

Gontar, 82.

Gontuad, 115.

Gotmar, 14, 41, 50.

Gotcefred, 117.

Guarengarda, 16.

Guifred, 4, 89, 99.

Guilia, 99.

Guillem, 60; -vescomte d'Empúries, 34.

Guillió, 64.

Guimarà, 22, 68, 88, 93, 116.

Guínidilda, 116.

Guisalmar, 49. 
Guisand, 45.

Guiscafred, 97.

Guisla, 65, 73, 116 .

Guisul, 72.

Guitard, 53, 68.

Gurb, castell i vila, 7 .

Hendelec, prevere, 71.

Hug, 43.

Indaleci, 29.

Ingilberga, abadessa, 15, 79.

Isarn, 18.

Issarn, 110.

«Karkases», 78.

Kasta, 50.

La Roca, 109.

Landeric, 60.

«Lasens», 77.

Ledgarda, 101; -monja, 102.

Leguard, 110.

Levegod, 67.

Lliçà d'Amunt, 89.

Llobatona, 78.

Llobet, 115.

Llobeta, 106.

Llobregat, 1.

Llorenç, 6, 25, 34, 63, 97.

«Lodares», 75.

«Lorint», 57.

Marca, 53.

Marcús, 1, 20.

Manresa, comtat, 54, 95, 110.

Maier, 94.

Manyanes, 111.

«Manyols», vila, 118.

Maria, 62, 86, 108.

«Marunyà), 29.

Mediona, 99. 
Miró, prevere, 113.

Mogent, 109.

Mogòria, 45.

Morató, 15, 63, $103,105$.

Morgades, 98.

Navarro, 10.

Negre, riu, 85.

Òdena, castell, 54.

Olèrdola, 12, 23, 24, 28.

Olibà, 11, 46, 48, 78; -suder, 94, 100.

«Olmedó», 5, 31.

Oló, castell, 95.

Onrada, 48, 89.

Or trobat, 1, 22, 36, 59, 80.

Oriol, sacerdot, 7.

Orrit, castell, 119.

Orsal, vila, 33,39 .

Orsalità, castell, 74, 75, 91, 96 .

Oruça, 4, 100.

Orús, 50, 109; -jutge, 36, 59, 108.

Osona, comtat, 3, 7, 15, 33, 39, 61, 74, 75, 77, 78, 79, 96, 98, 117.

Pallars, comtat, 85 .

Parets antigues, 90.

"Parets de Guisaliarda», 40.

Parets primes, 2, 36, 37, 38.

Pau, 6, 71.

Pedrer, 18.

Penyafel, 111.

Peralada, comtat, 79.

Pere, 48.

Perella, 97, 116.

«Perusa», 68.

Petró, 41.

Petroni, 15.

Pi, església, 48, 107.

Pinell, 53. 
Ponç Bliger, 118.

«Posticiosa», 95.

Prexat, 13.

Puig-Oriol, 74, 96.

Puig-reig, 82, 112.

«Pugnoso», vila, 20.

Provençals, vila, 1, 6, 9, 19, 26, 27, 34, 35, 37, 38, 41, 42,

$49,51,52,58,65,69,70,71,73,76,87,93$.

Quintuló, 106.

Quixel, 30.

Radulf, 33, 39; -Oriol, 119.

Ramon Berenguer, comte, 92.

Ramon [Borrell], comte, 3, 30, 43, 45, 93, I04; -comte [de

Pallars], 47, 119.

Ramon Folc, 92.

Ranló, 20.

Regiat, 87.

Regomir, 113.

Reifred, prevere, 112.

Ribagorça, comtat, 47.

Ribes, vall, 11, 46.

Ricarda, 79.

Rigol, 30.

Rihcell, 49.

Ripoll, vall, 15, 61, 78, 79, 98, 117.

Riquilda, 35, 43, 84, 90.

Roderad, 57.

Sabat, 108.

Saborida, 29.

Sala, 54, 56, 106.

Salamó, 18.

Sanç, 3, 35, 62, 72, 86; -prevere, 3; -Cortubí, 55.

Sant Adrià, terme, 16.

Sant Andreu, terme, 1, 22; -Apòstol, parròquia, 97.

Sant Joan de les Abadesses, monestir, 79, 102.

Sant Marti, casa de, 82; -[monestir], 99.

Sant Marti de Puig-reig, 112. 
Sant Pancraç, altar, 107.

Sant Pere Apòstol, església, 64.

Sant Pere de les Puel-les, cenobi, 22, 25, 93, 107.

Sant Pere de Riudebitlles, 99.

Sant Sadurní [de Tavèrnoles], 85.

Santa Maria, esglèsia, 48, cfr. Pi; -V. Tagamanent.

Santa Perpètua, castell, 106.

Sants, 55, 62, 72.

Segla, 14.

Sendra, 54.

Sendered, 24, 94.

Seniofred, 10, 84, 90, 91, 105; -vescomte, 33.

Seniol, 97.

Senior, 12.

Sentmenat, 101, 103, 105.

Sesenand, 36.

Sigirand, 55.

Sisuald, 37.

Sunla, 6 .

Sunyer, 34, 41.

Tagamanent, parròquia de Santa Maria, 92.

Tenes, vila, 79 .

Teofred, levita, 18.

Terraçola, 95, 99.

Terrassa, 60.

Teruntel, 32.

Teudebert, 48.

Teudelinda, abadessa, 93.

Teuderic, 21, 44.

Teudiscle, 83.

Tiano [Ticià, Sant Feliu de Llobregat], 32, 44, 66, 67.

Torre Blanca, 35, 36, 38, 84, 90.

Torre Franca, 54.

Torrelles, vall, 4 .

Torrent, lloc, 61, 117.

Tota, comtessa, 77.

Trasgonça, 8. 
Trasegòncia, 94.

Trasver, 116.

Tructelda, 8, 16, 44, 66.

Truitari, 95.

Trullols, 114.

Ugubert, 2, 9, 26; -levita, 19, 38, 40, 41, 42, 51, 52, 58, 65, $69,70,73,80$.

Unifred, 57.

Urgell, comtat, 85.

València, 1.

Valira, riu, 85 .

Vall-bovera, 83 .

Vallès, 5, 89, 101, 105, 109.

Vallespir, comtat, 102.

Vallfogona, 15.

Vallfornés, 92.

Vallmoll, 12, 23, 24, 28.

Veliard, 118.

Vila-Rubia, 33, 39.

Vilalba, 30, 104.

Vilea, 89.

Viscafred, 86.

Vives, $1,2,6,9,10,13,19,21,25,26,27,34,38,40,42$,

$49,52,56,58,63,65,69,70,71,73,76,81,84,88,90,97$, 116 ; - bisbe, 114. 